\title{
Is there a Role for the Appleby Procedure in 2020? Results from a Matched-Pair-Analysis
}

\author{
THOMAS MALINKA ${ }^{1 *}$, LEA TIMMERMANN $^{1 *}$, FRITZ KLEIN $^{1}$, \\ DOMINIK GEISEL ${ }^{2}$, JOHANN PRATSCHKE ${ }^{1}$ and MARCUS BAHRA ${ }^{1}$

\begin{abstract}
${ }^{1}$ Department of Surgery, Charité - Universitätsmedizin Berlin, corporate member of Freie Universität Berlin, Humboldt-Universität zu Berlin, and Berlin Institute of Health, Berlin, Germany;

${ }^{2}$ Department of Radiology, Charité - Universitätsmedizin Berlin, corporate member of Freie Universität Berlin, Humboldt-Universität zu Berlin, and Berlin Institute of Health, Berlin, Germany
\end{abstract}

\begin{abstract}
Background/Aim: Locally advanced pancreatic cancer (LAPC) of the pancreatic body involving the celiac axis requires specialized treatment, including a subtotal distal pancreatectomy (SDP) with resection of the celiac axis (Appleby procedure). This study aimed to examine the value of the Appleby procedure, in current individualized treatment approaches, and to define its possible therapeutic impact for patients with LAPC. Patients and Methods: 20 consecutive patients who underwent SDP with resection of the celiac axis between January 2005 and December 2018 were identified from a prospectively collected database and were matched with 20 patients experiencing SDP without resection of the celiac axis. Both perioperative parameters, as well as the overall postoperative course, were evaluated. Results: The rate of perioperative complications in both groups was comparable $(p=0.744)$. The rate of severe type $C$ postoperative pancreatic haemorrhages (PPH) was significantly lower in patients with resection of the celiac axis compared to those without ( $p=0.035)$. Conclusion: The Appleby procedure may be considered as a safe and feasible treatment option with favorably fewer postoperative severe bleeding complications. Besides surgical expertise, such procedures, however, require an experienced interventional radiologist and should thus only be performed in highvolume centers.
\end{abstract}

*Both Authors contributed equally to this manuscript.

Correspondence to: Thomas Malinka, Department of Surgery, Charité Campus Mitte and Charité Campus Virchow Klinikum, Charité Universitätsmedizin Berlin, Campus Virchow-Klinikum, Augustenburger Platz 1, 13353 Berlin, Germany. Tel: +49 30450552 001, e-mail: Thomas.Malinka@charite.de

Key Words: Distal pancreatectomy, Appleby procedure, outcome.
Systemic treatment options, such as neoadjuvant chemotherapy (NAC), are available for the treatment of pancreatic ductal adenocarcinoma (PDAC) and can improve overall survival rates. The only curative treatment strategy, however, still includes a radical surgical resection (1). Current definitions of resectability, defining PDAC subgroups of i) potentially resectable (RPC), ii) borderline resectable (BRPC) and iii) locally advanced types (LAPC), such as the NCCN guidelines for the definition of BRPC (2), mainly rely and focus on vessel involvement. Vessel involvement is nevertheless not always the limiting factor in therapeutic decision making, although it influences rsectability. PDAC of the pancreatic body is often associated with the involvement of the celiac axis (CA) as well as a late point of diagnosis. Symptoms are unspecific and mainly consist of back pain, weight loss, and unspecific abdominal pain (3). LAPC of the pancreatic body is associated with a reduced resection rate of $10 \%$ and a mean overall survival of 3-4 months (4). One possible treatment option, a subtotal distal pancreatectomy (SDP) with simultaneous CA resection, was initially described in 1976 (5). The first-ever illustrated resection of the celiac axis was performed in 1953 during an extended resection of gastric carcinoma and was named after the first surgeon to describe as the Appleby Procedure (6).

As the blood supply to the common hepatic artery (CHA) is maintained through collateral pathways over the gastroduodenal artery (GDA), and the stomach is supplied by the right gastric artery and gastroepiploic arteries fed by collaterals with the GDA over the superior mesenteric artery (SMA), resection of the CA has become a feasible treatment option for patients with advanced tumour infiltration. Favorable median survival times of 21 and 26 months have been described $(7,8)$. Nevertheless, liver necrosis and consecutive liver abscesses as well as gastric ulceration, necrosis, and perforation, have been described as the main procedure-related complications (9). Prior angiography with selective embolization of the CA can decrease the incidence 
of such complications by preoperatively conditioning the collateral pathways (10). To examine the value of the Appleby procedure, we performed a one-to-one matched-pair analysis with patients who underwent SDP without CA resection.

\section{Patients and Methods}

Data collection and matching criteria. Our retrospective singlecenter analysis was conducted at the Charité-Universitätsmedizin Berlin, Campus Virchow-Klinikum in Berlin, Germany. Data of all patients undergoing pancreatic resections between January 2005 and December 2018 were entered into a prospective database. All patients obtained a written informed consent, and an independent ethics committee approved the study design. Patients' characteristics were extracted from our medical records and further analyzed. All patients undergoing SDP with CA resection for PDAC were included. A one-to-one matched-pair analysis was performed by age, sex, and tumor entity with patients who underwent SDP without CA resection.

SDP was defined as a resection proximal to the superior mesenteric vessels. Patients undergoing distal pancreatectomy with a resection line distal to the superior mesenteric vessels as well as patients undergoing resection for other tumor entities were excluded.

Preoperative assessment, preconditioning, and surgical procedure. A multimodal therapeutic approach was individually formulated following initial diagnosis for every oncologic patient by a multidisciplinary tumor board, which consisted of medical oncologists, specialized radiation therapists, radiologists, and surgeons. Multiphase computed tomography (CT), or magnetic resonance imaging (MRI) with contrast agents were routinely used for radiological diagnostics and planning. An MRI showing an encased CA is shown in Figure 1.

Patients were excluded from exploration in the presence of widespread metastatic disease. Preoperatively, the clinical diagnostic tests included standard physical examination and routine laboratory testing as well as measuring CA19-9 serum levels. When preoperative CT or MRI showed tumor involvement of the CA and CHA but no impairment of the SMA and GDA, we evaluated patients for an Appleby procedure. Preoperative embolization of the $\mathrm{CA}$ and the CHA was executed if further diagnostics revealed appropriate conditions for resection without distant metastasis. All patients in our case group underwent preresectional embolization. After preparation, a selective angiography was performed. CA and SMA were catheterized simultaneously and following a temporary blocking of the CHA with a balloon, digital subtraction angiography of the SMA was performed to demask proper collateralization. If collateralization was judged as appropriate, coil or Amplatzer vascular plug occlusions of the CHA, left gastric artery and splenic artery as well as - if technically feasible - CA were performed. With this, the direct arterial blood supply from the CA to the liver and stomach was interrupted. Through collateral pathways over the pancreatoduodenal arcade to the GDA, the arterial blood supply was enhanced to the proper hepatic artery (PHA) and the gastroepiploic artery, as previously described (10). Radiographic imaging of balloon occlusion, coiling and collateralization are demonstrated in Figures 2-4. Following a short in-hospital stay, patients were sent home and returned for subsequent resection.
Experienced surgeons performed all procedures according to international standards at that time. Somatostatin analogs were not prescribed. Dissection of the pancreas was either done using electrocautery or a stapling device. In cases of pancreatic resections performed by electrocautery, a subsequent closure of the main pancreatic duct of the pancreatic remnant was achieved by a stitch ligation using 4-0 polypropylene sutures, followed by single Ushaped 4-0 polypropylene sutures (Prolene, Johnson \& Johnson Medical GmbH, Norderstedt, Germany). Distal closure of the pancreatic remnant by stapler was performed using linear stapling devices armed with a black $60-\mathrm{mm}$ cartridge (EndoGIA ${ }^{\mathrm{TM}}$, AutoSuture, Covidien). Cartridges were reinforced by a bioabsorbable mesh (SEAMGUARD®, W.L. Gore, Flagstaff, AZ, USA). CAresection was secured using both ligature and suture in all open surgery cases. In the case of robotic-assisted surgery a vascular stapler was used. All included patients underwent a simultaneous splenectomy and received at least one intra-abdominal drain (Degania Silicone Europe GmbH, Regensburg, Germany) to measure the postoperative lipase levels, while the drain output was placed directly at the pancreatic resection line.

Postoperative evaluation. Following surgery, all patients were monitored at a specialized surgical intensive care unit for at least one day. The lipase levels were repeatedly assessed and measured in the serum and in the intraoperatively placed abdominal drains to identify postoperative pancreatic fistula. In the absence of signs of POPF and DGE, oral food intake was started depending on the clinical presentation and tolerance. The Clavien-Dindo (CD) classification was used to grade postoperative complications (11). Major complications were defined as $\mathrm{CD} \geq 3 \mathrm{a}$. All resected specimens were histologically examined to identify the underlying tumor entity and completeness of resection by an attending pathologist. Postoperative application of chemotherapy and radiotherapy was considered case-by-case, based on factors, such as i) the completeness of the resection, ii) local histopathological tumor stage, iii) response to prior therapy, and iv) the patient's condition.

Data collection. Collected and reviewed data included preoperative ASA, BMI, demographics, time from embolization to resection, application of neoadjuvant chemotherapy as well as postoperative local histopathological tumor stage (TNM) and infiltration of resection margins. Furthermore, postoperative morbidity and mortality were recorded: POPF, PPH, SSI, DGE, in-hospital stay, ICU-stay, the requirement of reoperation or intervention, mortality within 30 days, and readmission within 90 days.

Statistics. Statistics present mean (range) or numbers (\%). Twotailed Pearson's chi-square test and Friedman-test were used to compare ordinal or nominal scaled variables, respectively. Significance tests were two-sided, and $p<0.05$ was considered asstatistically significant. All statistical analyses, as well as initial matching of cases and controls, were performed using SPSS, version 25.0 (SPSS, Chicago, IL, USA).

\section{Results}

Patient characteristics. An overall of 40 patients was included in this study. Twenty patients underwent SDP with additional resection of the $\mathrm{CA}$, and the other 20 patients 


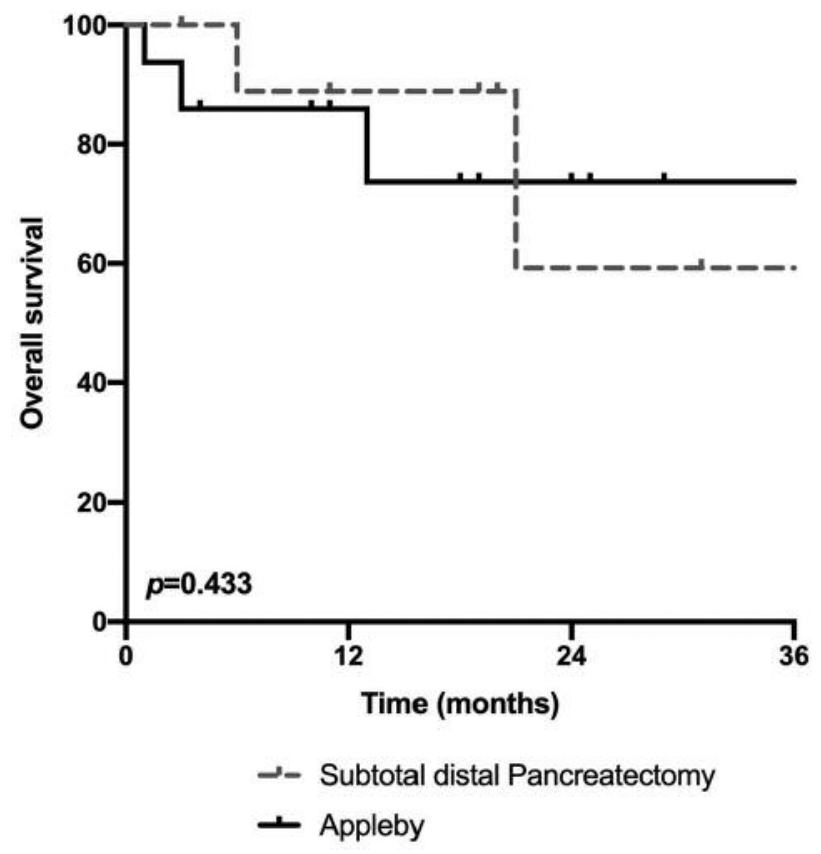

Figure 1. Survival curves. Overall survival (OS) for patients undergoing subtotal distal pancreatectomy with resection of thenceliac axis (Appleby) and for patients undergoing subtotal distal pancreatectomy without resection of the celiac axis with no significant difference $(p=0.433)$.

experienced SDP without resection of the CA. Out of all the patients, $45 \%$ were female, and $55 \%$ male. The mean age was 62.9 years (37-79 years). Preoperative ASA scores and BMI were comparable. One patient underwent SDP with simultaneous CA resection with the use of the DaVinci robotic system. Table I indicates patient characteristics.

Perioperative parameters. The postoperative histopathological tumor stage was comparable in both groups (Table II). The most common T stage was T3 (69.2\%), while nodal positivity was found in $55 \%$ of the patients in our case group and in $50 \%$ of the patients in the control group $(p=0.949)$. The largest tumor diameter was comparable between the two groups as well, with a mean of $30.94 \mathrm{~mm}$ (18-59) in the case group and $27.9 \mathrm{~mm}(12-56)$ in the control group $(p=0.443)$. From the case group, $25 \%$ of the patients underwent neoadjuvant treatment with either FOLFIRINOX or Gemcitabine/nabPaclitaxel compared to $20 \%$ of the patients from the control group. The same amount $(25 \%)$ of patients in the case group, went under an additional portal vein resection, whereas in our control group, only 5\% underwent an additional portal vein resection. Direct anastomosis or a patch plastic using a falciform ligament, VMI patch or Vascu Guard Patch achieved vascular reconstruction. Additional partial gastric resections were performed in $10 \%$ of the patients in our case group and in $20 \%$ of the patients in our
Table I. Patient characteristics.

\begin{tabular}{lcccc}
\hline $\begin{array}{l}\text { Characteristics } \\
\mathrm{N}(\%)\end{array}$ & $\begin{array}{c}\text { All } \\
\text { patients } \\
(\mathrm{N}=40)\end{array}$ & $\begin{array}{c}\text { Appleby } \\
\text { procedure } \\
(\mathrm{N}=20)\end{array}$ & $\begin{array}{c}\text { Subtotal distal } \\
\text { pancreatectomy } \\
(\mathrm{N}=20)\end{array}$ & $p$-Value \\
\hline Gender N (\%) & & & & 1.0 \\
Male & $22(55)$ & $11(55)$ & $11(55)$ & \\
Female & $18(45)$ & $9(45)$ & $9(45)$ & 0.37 \\
Age (years) & & & & \\
Mean & 62.9 & 60.75 & 65.05 & \\
Minimum & 37 & 37 & 46 & \\
Maximum & 79 & 77 & 79 & 0.384 \\
ASA Score N (\%) & & & & \\
1 & $4(10)$ & $3(15)$ & $1(5)$ & \\
2 & $21(52.5)$ & $8(40)$ & $13(65)$ & \\
3 & $10(25)$ & $4(12.5)$ & $6(30)$ & \\
4 & $0(0)$ & $0(0)$ & $0(0)$ & \\
BMI N (\%) & & & & \\
Mean & 25.7 & 25.5 & 25.9 & \\
Minimum & 19.8 & 19.8 & 19.8 & \\
Maximum & 36.9 & 35.5 & 36.9 & \\
\hline
\end{tabular}

ASA: American Society of Anesthesiologists; BMI: body mass index.

control group. In our case group, resection was performed after a mean of 11.2 (4-20) days after selective angiography and embolization.

SDP including CA resection with the use of a robotic system. One patient in our case group, a 58-year-old male without any prior abdominal operations, underwent resection using the DaVinci robotic system six days after embolization. No further vessel resections were necessary. He underwent NAC with FOLFIRINOX before embolization and resection. The tumour diameter was $55 \mathrm{~mm}$, and the histopathological TNM stage was ypT3 ypN1 G2 R0 L0 V0. The in-hospital stay was nine days, including one day in the intensive care unit. No postoperative complications occured during the inhospital stay, and no readmission was necessary.

Complications. Overall, complications were comparable in both groups. In our case group a rate of $60 \%$ overall complications, and in our control group a rate of $65 \%$ overall complications appeared $(p=0.744)$. Significant complications, classified as $\mathrm{CD} \geq 3 \mathrm{a}$, appeared in $50 \%$ of the patients in our case group and in 55\% of the patients in our control group. Complications, such as POPF, SSI, DGE, and others, including cardiovascular, pulmonary, and urinary tract complications, appeared in a comparable number in both groups (Table III). However, as the overall appearance of PPH was similar in both groups $(p=0.1)$, the severity was significantly higher in the control compared to the case group, with no type $\mathrm{C}$ bleeding in our case group and four type $\mathrm{C}$ 
Table II. Perioperative parameters.

\begin{tabular}{|c|c|c|c|c|}
\hline Postoperative T-Stage N (\%) & & & & 0.443 \\
\hline $\mathrm{T} 1$ & $5(12.8)$ & $1(10.5)$ & $3(15)$ & \\
\hline $\mathrm{T} 2$ & $5(12.8)$ & $3(15.8)$ & $2(10)$ & \\
\hline $\mathrm{T} 3$ & $27(69.2)$ & $12(63.2)$ & $15(75)$ & \\
\hline $\mathrm{T} 4$ & $2(5.1)$ & $2(10.5)$ & $0(0)$ & \\
\hline Postoperative N-Stage N (\%) & & & & 0.949 \\
\hline No & $19(47.5)$ & $9(45)$ & $10(50)$ & \\
\hline $\mathrm{N} 1$ & $19(47.5)$ & $10(50)$ & $9(45)$ & \\
\hline $\mathrm{N} 2$ & $2(5)$ & $1(5)$ & $1(5)$ & \\
\hline Postoperative L-Stage N (\%) & & & & 0.736 \\
\hline L0 & $27(67.5)$ & $14(70)$ & $13(65)$ & \\
\hline L1 & $13(32.5)$ & $6(30)$ & $7(35)$ & \\
\hline Postoperative V-Stage N (\%) & & & & 0.311 \\
\hline V0 & $27(67.5)$ & $12(60)$ & $15(75)$ & \\
\hline V1 & $13(32.5)$ & $8(40)$ & $5(25)$ & \\
\hline Tumour Diameter (mm) & & & & 0.443 \\
\hline Mean & 29.34 & 30.94 & 27.9 & \\
\hline Minimum & 12 & 18 & 12 & \\
\hline Maximum & 59 & 59 & 56 & \\
\hline Grading N (\%) & & & & 0.691 \\
\hline G1 & $3(7.7)$ & $1(5.3)$ & $2(10)$ & \\
\hline $\mathrm{G} 2$ & $25(64.1)$ & $13(68.4)$ & $12(60)$ & \\
\hline G3 & $11(28.2)$ & $5(26.3)$ & $6(30)$ & \\
\hline Postoperative resection stage $\mathrm{N}(\%)$ & & & & 0.342 \\
\hline R0 & $19(47.5)$ & $8(40)$ & $11(55)$ & \\
\hline $\mathrm{R} 1$ & $21(52.5)$ & $12(60)$ & $9(45)$ & \\
\hline Neoadjuvant treatment $\mathrm{N}(\%)$ & $9(22.5)$ & $5(25)$ & $4(20)$ & 0.705 \\
\hline \multicolumn{5}{|l|}{ Additional vessel resection N (\%) } \\
\hline Portal vein resection & $6(15)$ & $5(25)$ & $1(5)$ & 0.212 \\
\hline
\end{tabular}

bleedings in our control group ( $p=0.035)$. In our case group, two patients developed gastric complications, including a gastric perforation and gastric ischemia with the need for reoperation. There was no 30-day mortality in our case group, but there was an in-hospital mortality within 30 days in two cases of our control group ( $p=0.147)$. The in-hospital stay was comparable in both groups, with a mean of 29.45 days (7-162) in our case group and 31.65 days (11-93) in our control group ( $p=0.409)$ (Table III). Survival curves for both groups did not reveal any significant differences between the groups $(p=0.433$; Figure 1).

\section{Discussion}

LAPC of the pancreatic body has a sparse prognosis and is described with a resectability rate of $10 \%$ and a mean survival of 3-4 months (4). The ability of additional CA resections in the case of CA involvement has led to a favorable median survival of 24.1 months following resection (12).

Pain release after resecting the infiltrated retroperitoneal nerval plexus may lead to an additional improvement of life quality (8). SDP with additional CA resection appears to be a safe treatment option with a comparable overall complication
Table III. Complications.

\begin{tabular}{|c|c|c|c|c|}
\hline $\begin{array}{l}\text { Characteristics } \\
\mathrm{N}(\%)\end{array}$ & $\begin{array}{c}\text { All } \\
\text { patients } \\
(\mathrm{N}=40)\end{array}$ & $\begin{array}{l}\text { Appleby } \\
\text { procedure } \\
(\mathrm{N}=20)\end{array}$ & $\begin{array}{l}\text { Subtotal distal } \\
\text { pancreatectomy } \\
\quad(\mathrm{N}=20)\end{array}$ & $p$-Value \\
\hline \multicolumn{5}{|l|}{ Overall complications } \\
\hline $\mathrm{N}(\%)$ & $25(62.5)$ & $12(60)$ & $13(65)$ & 0.744 \\
\hline \multicolumn{5}{|l|}{$\begin{array}{l}\text { Clavien/Dindo } \\
\text { classification N (\%) }\end{array}$} \\
\hline 0 & $14(35)$ & $8(40)$ & $6(30)$ & \\
\hline 1 & $3(7.5)$ & $1(5)$ & $2(10)$ & \\
\hline 2 & $2(5)$ & $1(5)$ & $1(5)$ & \\
\hline $3 \mathrm{a}$ & $8(20)$ & $6(30)$ & $2(10)$ & \\
\hline $3 b$ & $5(12.5)$ & $2(10)$ & $3(15)$ & \\
\hline $4 a$ & $4(10)$ & $0(0)$ & $4(20)$ & \\
\hline $4 b$ & $1(2.5)$ & $1(5)$ & $0(0)$ & \\
\hline 5 & $3(7.5)$ & $1(5)$ & $2(10)$ & \\
\hline POPF N (\%) & & & & 0.619 \\
\hline Biochemical leak & $3(7.5)$ & $1(5)$ & $2(10)$ & \\
\hline B & $16(40)$ & $7(35)$ & $9(45)$ & \\
\hline $\mathrm{C}$ & $1(2.5)$ & $1(5)$ & $0(0)$ & \\
\hline PPH N (\%) & & & & 0.1 \\
\hline A & $0(0)$ & $0(0)$ & $0(0)$ & \\
\hline B & $3(7.5)$ & $2(10)$ & $1(5)$ & \\
\hline $\mathrm{C}$ & $4(10)$ & $0(0)$ & $4(20)$ & 0.035 \\
\hline SSI N (\%) & $2(5)$ & $1(5)$ & $1(5)$ & 1.0 \\
\hline DGE N (\%) & $1(2.5)$ & $1(5)$ & $0(0)$ & 0.311 \\
\hline \multicolumn{5}{|l|}{ Other complications } \\
\hline $\mathrm{N}(\%)$ & $11(27.5)$ & $5(25)$ & $6(30)$ & 0.723 \\
\hline Gastric ischemia & $1(2.5)$ & $1(5)$ & $0(0)$ & \\
\hline Gastric perforation & $1(2.5)$ & $1(5)$ & $0(0)$ & \\
\hline \multicolumn{5}{|l|}{ Reoperation rate } \\
\hline $\mathrm{N}(\%)$ & $8(20)$ & $3(15)$ & $5(25)$ & 0.429 \\
\hline \multicolumn{5}{|l|}{ 30-day mortality } \\
\hline $\mathrm{N}(\%)$ & $2(5)$ & $0(0)$ & $2(10)$ & 0.147 \\
\hline $\begin{array}{l}\text { 90-day readmission } \\
\text { rate } \mathrm{N}(\%)\end{array}$ & $6(15)$ & $3(15)$ & $3(15)$ & 1.0 \\
\hline Inhospital stay (days) & & & & 0.409 \\
\hline Minimum & 7 & 7 & 11 & \\
\hline Maximum & 162 & 162 & 93 & \\
\hline Mean & 30.55 & 29.45 & 31.65 & \\
\hline ICU stay (days) & & & & 0.281 \\
\hline Minimum & 1 & 1 & 1 & \\
\hline Maximum & 130 & 130 & 81 & \\
\hline Mean & 13.05 & 10.3 & 15.8 & \\
\hline
\end{tabular}

POPF: Postoperative pancreatic fistula; PPH: postoperative pancreatic haemorrhage; SSI: surgical site infection; DGE: delayed gastric emptying; ICU: intensive care unit.

rate and rate of major complications $(C D \geq 3 a)$ between our case and control cohort. Mortality and morbidity of patients undergoing SDP with CA resection have been described with $0 \%$ and $47.1 \%, 2.1 \%$ and $40.6 \%$, and $0 \%$ and $62 \%$, respectively in three studies, comparable with $0 \%$ and $60 \%$ found in our study $(8,13,9)$. PPH is one of the most common and threatening complications in pancreatic surgery and one of the most important reasons for reoperation and mortality. The most common bleeding source in cases of SDP is the 
splenic artery $(14,15)$. In our case group, following embolization and CA resection, no $\mathrm{PPH}$ type $\mathrm{C}$ were observed, whereas four type $\mathrm{C}$ bleedings occurred in our control group. The Appleby procedure appears to decrease the risk of severe PPH significantly. A combination of embolization and consecutive $\mathrm{CA}$ resection may serve as an explanation. After resection of the nerval plexus, a higher incidence of postoperative and persistent diarrhea has been described (16). In our cohort, no increased incidence of diarrhea appeared; postoperative diarrhea was mainly caused by transient or persistent exocrine insufficiency and was successfully treated with the substitution of pancreatic enzymes.

Current definitions of PDAC subtypes are based on vessel involvement or encasement, and mainly rely on anatomical rather than on clinically relevant parameters (17). The Appleby procedure may serve as an example for the need of redefining the PDAC subtypes and the resectability criteria as it is a safe treatment option with favorable survival rates. In our cohort, patients both undergoing or not undergoing $\mathrm{CA}$ resection had comparable survival rates. If indicated, CA resection should, thus, be performed regardless of anatomically defined classifications. Typical complications related to CA resection are gastric or hepatic ischemia. Gastric ischemia has been described to occur in $12.87 \%$ of all patients, whereas prior embolization can lead to a favorable incidence of $10.74 \%$ compared to $14.38 \%$ in cases without prior embolization (12). Our case cohort had a comparable frequency of 5\% gastric ischemia and 5\% gastric perforation in all patietns undergoing prior selective embolization. Resectability can, in each patient, only be finally evaluated during exploration. This is even enhanced by the fact that regression following NAC can be hardly distinguished radiographically from vital tumor tissues. Therefore, embolization can, from time to time, be done unnecessarily (18).

PDAC, however, should be considered as a systemic disease, with the main life-threatening issues being recurrence and metastasis (8). In our cohort, we found a rate of $\mathrm{R} 0$ resections of $40 \%$ in our case group and $55 \%$ in our control group. A meta-analysis has previously found a R0 rate of $72.79 \%$ (30.8-100\%) (12). Performing the Appleby procedures following neoadjuvant chemotherapy has resulted in an $\mathrm{R} 0$ resection rate of $91 \%$ (18). Infiltrated resection margins can be correlated with the deterioration of prognosis, whereas NAC is correlated with a higher amount of R0 resections (19). Resectability of LAPC of the pancreatic body with infiltration of the CA is often limited by simultaneous SMA infiltration, because both vessels cannot be resected at the same time (20).

Another point in the current field of LAPC treatment is the use of laparoscopy and robotic systems. In our case, the use of a robotic system was a feasible treatment option. Its value in the individualized treatment of LAPC, however, needs further assessment.
The strengths of our study include: i) the case-matched study design, and ii) the homogeneity within both groups. The present study is limited by common biases that are mainly due to the retrospective character of this analysis. Precision and completeness of data acquisition are tough to control, especially over such an extended study period. To encounter this condition, we used a carefully matched control group.In conclusion, CA resection in patients with LAPC of the pancreatic body appears to be a safe treatment option increasing a favorable outcome with few severe bleeding complications. It, nevertheless, requires an experienced interdisciplinary treatment team and should thus only be performed at a high volume centre. At times of individualized treatment approaches tailored to the patient's needs, NAC appears to further improve R0 resection rates and survival time. Also, the use of minimally invasive treatment options, such as the use of a robotic system in the treatment of LAPC, needs further assessment in prospective trials.

\section{Conflicts of Interest}

All Authors declare no commercial associations that might represent conflicts of interest with this article and have nothing to disclose.

\section{Authors' Contributions}

Substantial contributions to the conception or design of the study were done by TM, LT, FK, DG, JP, MB. Acquisition, analysis, or interpretation of data for the study was done by TM, LT, FK, JP, MB. Drafting the work or revising it critically for intellectual content was done by TM, LT, FK, DG, JP, MB. Final approval of the version to be published was done by TM, LT, FK, DG, JP, MB.

\section{References}

1 Buanes TA: Role of surgery in pancreatic cancer. World J Gastroenterol 23(21): 3765-3770, 2017. PMID: 28638216. DOI: 10.3748/wjg.v23.i21.3765

2 Bockhorn M, Uzunoglu FG, Adham M, Imrie C, Milicevic M, Sandberg AA, Asbun HJ, Bassi C, Büchler M, Charnley RM, Conlon K, Cruz LF, Dervenis, C, Fingerhutt A, Friess H, Gouma DJ, Hartwig W, Lillemoe KD, Montorsi M, Neoptolemos JP, Shrikhande SV, Takaori K, Traverso W, Vashist YK, Vollmer C, Yeo CJ and Izbicki JR: Borderline resectable pancreatic cancer: a consensus statement by the International Study Group of Pancreatic Surgery (ISGPS). Surgery 155(6): 977-988, 2014. PMID: 24856119. DOI: 10.1016/j.surg.2014.02.001

3 Wang X, Dong Y, Jin J, Liu Q, Zhan Q, Chen H, Shen B, Deng $\mathrm{X}$, Pang $\mathrm{C}$ and Li H: Efficacy of modified Appleby surgery: a benefit for elderly patients? J Surg Res 194(1): 83-90, 2015. PMID: 25311939. DOI: 10.1016/j.jss.2014.09.014

4 Nordback IH, Hruban RH, Boitnott JK, Pitt HA and Cameron JL: Carcinoma of the body and tail of the pancreas. Am J Surg 164(1): 26-31, 1992. PMID: 1378243. DOI: 10.1016/s00029610(05)80641-5 
5 Nimura Y HT, Miura K, Nakajima N and Hibi M: Experience of Appleby's operation for advanced carcinoma of the pancreatic body and tail. Shujutsu 30: 885-889, 1976.

6 Appleby LH: The coeliac axis in the expansion of the operation for gastric carcinoma. Cancer 6(4): 704-707, 1953. PMID: 13059764. DOI: 10.1002/1097-0142(195307)6:4<704::aidcncr2820060410>3.0.co;2-p

7 Hirano S, Kondo S, Hara T, Ambo Y, Tanaka E, Shichinohe T, Suzuki O and Hazama K: Distal pancreatectomy with en bloc celiac axis resection for locally advanced pancreatic body cancer: long-term results: Ann Surg 246(1): 46-51, 2007. PMID: 17592290. DOI: 10.1097/01.sla.0000258608.52615.5a

8 Shen Q, Jiang QF, Tian YW, Yu M, Jia JK and Xue HZ: Appleby operation for carcinoma of the body and tail of the pancreas. $J$ Cancer Res Ther 14(Suppl): 1019-1023, 2018. PMID: 30539839. DOI: $10.4103 / 0973-1482.199383$

9 Kondo S, Katoh H, Hirano S, Ambo Y, Tanaka E, Okushiba S and Morikawa T: Results of radical distal pancreatectomy with en bloc resection of the celiac artery for locally advanced cancer of the pancreatic body. Langenbecks Arch Surg 388(2): 101-106, 2003. PMID: 12684805. DOI: 10.1007/s00423-003-0375-5

10 Denecke T, Andreou A, Podrabsky P, Grieser C, Warnick P, Bahra M, Klein F, Hamm B, Neuhaus P and Glanemann M: Distal pancreatectomy with en bloc resection of the celiac trunk for extended pancreatic tumor disease: an interdisciplinary approach. Cardiovasc Intervent Radiol 34(5): 1058-1064, 2011. PMID: 20936285. DOI: 10.1007/s00270-010-9997-5

11 Dindo D, Demartines N and Clavien PA: Classification of surgical complications: a new proposal with evaluation in a cohort of 6336 patients and results of a survey. Ann Surg 240(2): 205-213, 2004. PMID: 15273542. DOI: 10.1097/01.sla.0000133083.54934.ae

12 Gong H, Ma R, Gong J, Cai C, Song Z and Xu B: Distal Pancreatectomy With En Bloc Celiac Axis Resection for Locally Advanced Pancreatic Cancer: A Systematic Review and MetaAnalysis. Medicine (Baltimore) 95(10): e3061, 2016. PMID: 26962836. DOI: 10.1097/MD.000000000003061

13 Sperti C, Berselli M and Pedrazzoli S: Distal pancreatectomy for body-tail pancreatic cancer: is there a role for celiac axis resection? Pancreatology O(4): 491-498, 2010. PMID: 20720451. DOI: $10.1159 / 000276984$

14 Izumo W, Higuchi R, Yazawa T, Uemura S, Shiihara M and Yamamoto M: Evaluation of preoperative risk factors for postpancreatectomy hemorrhage. Langenbecks Arch Surg, 2019. PMID: 31650216. DOI: 10.1007/s00423-019-01830-w
15 Feng F, Cao X, Liu X, Qin J, Xing Z, Duan J, Liu C and Liu J: Two forms of one complication: Late erosive and nonerosive postpancreatectomy hemorrhage following laparoscopic pancreaticoduodenectomy. Medicine (Baltimore) 98(30): e16394. PMID: 31348239. DOI: 10.1097/MD.0000000000016394

16 Hirano S, Kondo S, Tanaka E, Shichinohe T, Tsuchikawa T, Kato $\mathrm{K}$ and Matsumoto $\mathrm{J}$ : Postoperative bowel function and nutritional status following distal pancreatectomy with en-bloc celiac axis resection. Dig Surg 27(3): 212-216, 2010. PMID: 20571268. DOI: 10.1159/000265573

17 Tempero MA, Malafa MP, Chiorean EG, Czito B, Scaife C, Narang AK, Fountzilas C, Wolpin BM, Al-Hawary M, Asbun H, Behrman SW, Benson AB, Binder E, Cardin DB, Cha C, Chung V, Dillhoff M, Dotan E, Ferrone CR, Fisher G, Hardacre J, Hawkins WG, Ko AH, LoConte N, Lowy, AM, Moravek C, Nakakura EK, O'Reilly EM, Obando J, Reddy S, Thayer S, Wolff RA, Burns JL and Zuccarino-Catania G: Pancreatic Adenocarcinoma, Version 1.2019. J Natl Compr Canc Netw 17(3): 202-210, 2019. PMID: 30865919. DOI: 10.6004/jnccn.2019.0014

18 Baumgartner JM, Krasinskas A, Daouadi M, Zureikat A, Marsh W, Lee K, Bartlett D, Moser AJ and Zeh HJ: Distal pancreatectomy with en bloc celiac axis resection for locally advanced pancreatic adenocarcinoma following neoadjuvant therapy. J Gastrointest Surg 16(6): 1152-1159, 2012. PMID: 22399269. DOI: 10.1007/s11605-012-1839-0

19 Dhir M, Malhotra GK, Sohal DPS, Hein NA, Smith LM, O'Reilly EM, Bahary N and Are C:Neoadjuvant treatment of pancreatic adenocarcinoma: a systematic review and metaanalysis of 5520 patients. World J Surg Oncol 15(1): 183, 2017. PMID: 29017581. DOI: 10.1186/s12957-017-1240-2

20 Hishinuma S, Ogata Y, Tomikawa M and Ozawa I: Stomachpreserving distal pancreatectomy with combined resection of the celiac artery: radical procedure for locally advanced cancer of the pancreatic body. J Gastrointest Surg 11(6): 743-749, 2007. PMID: 17417712. DOI: 10.1007/s11605-007-0143-x

Received November 30, 2019

Revised December 10, 2019

Accepted December 13, 2019 